\title{
LIVER SEGMENTATION FROM CT IMAGES USING A MODIFIED DISTANCE REGULARIZED LEVEL SET MODEL BASED ON A NOVEL BALLOON FORCE
}

\author{
Nuseiba M. Altarawneh ${ }^{1}$, SuhuaiLuo ${ }^{1}$, Brian Regan ${ }^{1}$, Changming Sun $^{2}$ \\ ${ }^{1}$ School of Design Communication and IT, \\ The University of Newcastle, Callaghan NSW 2308, Australia \\ ${ }^{2}$ Computational Informatics, CSIRO, North Ryde, NSW 1670, Australia \\ nuseiba.altarawneh@uon.edu.au, suhuai.luo@newcastle.edu.au, \\ brian.reganenewcastle.edu.au, changming.sundcsiro.au
}

\begin{abstract}
Organ segmentation from medical images is still an open problem and liver segmentation is a much more challenging task among other organ segmentations. This paper presents a liver segmentation method from a sequence of computer to mography images. We propose a novel balloon force that controls the direction of the evolution process and slows down the evolving contour in regions with weak or without edges and discourages the evolving contour from going far away from the liver boundary or from leaking at a region that has a weak edge, or does not have an edge. The model is implemented using a modified Distance Regularized Level Set (DRLS) model. The experimental results show that the method can achieve a satisfactory result. Comparing with the original DRLS model, our model is more effective in dealing with over segmentation problems.
\end{abstract}

\section{KEYWORDS}

Liver segmentation, level set method, Distance Regularized Level Set (DRLS) model

\section{INTRODUCTION}

The liver is one of the most important organs in the human body. It carries out a variety of functions including filtering the blood, making bile and proteins, processing sugar, breaking down medications, and storing iron, minerals and vitamins. However, the liver is prone to many diseases such as hepatitis $\mathrm{C}$, cirrhosis, and cancer. As the advance of computer science and technology, computer-aided surgical planning systems (CAD) have played an important role in diagnosing and treatment of liver diseases. These systems can present the structures of various liver vessels, generate resection proposals, offer 3D visualizations, provide surgical simulations with cutting, and lead to shorter planning times. However, among these systems, one of the most 
important problems is the accurate segmentation of a liver from its surrounding organs in computer tomography (CT) images.

Developing a robust method for liver segmentation from CT images is a challenging task due to the similar intensity values between adjacent organs, geometrically complex liver structure and the injection of contrast media, which causes all tissues to have different gray level values. Several artefacts of pulsation and motion, and partial volume effects also increase the difficulties to carry out automatic liver segmentation in CT images. The significant variations in shape and volume of the liver also contribute these difficulties. Therefore, liver segmentation from medical images is still an open problem. Generally, methods and approaches to liver segmentation in the CT images are categorized into two main categories: semiautomatic and fully automatic liver segmentation methods. Semi-automatic liver segmentation methods require a limited user intervention to complete the task. This intervention varies from a manual selection for seed points to a manual refinement of a binary mask for the liver. The term fully automated means that the liver segmentation process is implemented without any sort of operator intervention. This kind of method is highly appreciated by radiologists since it is free from user errors and biases, and it saves the operator from a potentially hard work and wasted time. The latest achievements in liver segmentation are reviewed in this section. All the methods are discussed in one of the three categories; namely, gray level based methods, model based methods, and texture based methods [1]. It is apparent that each category of liver segmentation methods has its own advantages and shortcomings, and may be effective for a particular case. With the gray level based methods[211], they use image feature directly. Consequently, gray level based methods are the most common methods in liver segmentation.

These methods depend primarily on the evolution of gray level toward targets. While gray level methods are generally fast, their effectiveness may be affected, especially when gray level intensity of targets exhibits changes. In spite of using prior knowledge, gray level methods may fail when the liver occupies a small percentage of the image. Gray level is applied manually or via automatic rough segmentation. The aim of these two procedures is to collect information with regard to the gray level. Whilst these methods are reliable, they often require substantial computational time. Several gray level based methods deploy gradient information as a precise approach to deal with image boundaries. However, this approach becomes impractical in the presence of numerous boundaries, only some of them are the real boundaries of the desired object. Under these conditions, gray level based methods may easily converge to wrong boundaries, resulting in over or under segmentation. This could be corrected by refining the results through a manual work or via the implementation of other methods.

In contrast, structure based methods [12-15] are capable of dealing with unclear liver boundaries by utilising prior knowledge. Structure based methods can handle some problems which the gray level based methods cannot deal with. However, these methods require a great deal of training data to span all plausible conditions of the liver. Applying these methods incur significant difficulty when handling nonstandard shapes for the liver. In other words, it is very hard to develop a unified segmentation models for liver based on structure based methods. Instead of using gray level or shapes, texture based methods [16-19]utilises pattern recognition and machine learning to locate boundaries. As a result, these methods enable one to collectively consider more features. Texture based methods can produce better results when the boundaries are not clear. An accurate description of texture feature constitutes the main challenges in these methods in addition to the need for training data. Although there exist many descriptors, they are not like those described by human. Furthermore, selecting a proper descriptor out of many, poses another 
problem. It should be noted that both machine learning and pattern recognition are still developing technologies with much weaker information processing abilities than human brain and they are not able to achieve good segmentation result without the use of other refined methods. In general, gray level based methods are more highly developed. They can conveniently be used to deal with complex segmentation tasks.

In the majority of cases, gray level based methods can attain satisfactory segmentation results. Structure based methods relies heavily on the shape of the object, a property making them a more robust technique. Finally, texture based methods attempt to emulate the procedure that human's brain processes. Level set methods have been investigated and widely utilized in image segmentation especially for medical images segmentation[20-22].Current approaches in using level set methods represent promising approaches for segmenting irregular object shapes such as liver. However it has a strict requirement on the initial position. It achieves a good result when the initial contour is placed near the target.3D liver segmentation methods can be categorized into two classes: direct 3D segmentation and propagation of the 2D slice-based segmentation. In terms of the first class, the user initializes a 3D deformable surface in multiple 2D slices of the liver, and then the initial 3D mesh is automatically refined by forces characterized by the image gradient and smoothness of the contour. This kind of method is time consuming and requires many user interactions that can lead to observer variability. The second class of 3D liver segmentation makes use of the slice-based propagation approach. In this technique, the 3D CT images are re-sliced into a number of 2D slices. A 2D segmentation is used in each slice, which is initialized by a propagated boundary from the previous $2 \mathrm{D}$ slice. This technique reduced a $3 \mathrm{D}$ segmentation problem to a sequence of $2 \mathrm{D}$ segmentation problems. Each of the reduced $2 \mathrm{D}$ segmentation sub-problems is much simpler than the original 3D segmentation problem, and it is also much cheaper on computational expense to incorporate 2D shape information as a shape constraint into the 3D segmentation procedure. Since the difference between adjacent slices is small, the final contour of one slice can provide useful information about the initial contour position and prior intensity and shape information which in turn enhances the segmentation performance of the level set method for the following slices. In this paper, we will modify the distance regularization level set [23] (DRLS) model in order to segment the liver contour in each 2D slice by using a new balloon forces that controls the direction of the evolution and slows down the evolution process in the region with weak or without edges. This papers organized as follows: Section 2 reviews the DRLS model. Section3 describes our methodology. Section4 shows some experimental results, and finally the conclusion is conveyed in Section 5.

\section{DISTANCE REGULARIZED LEVEL SET METHOD}

Liet al.[23]proposed a level set method termed as Distance Regularized Level Set (DRLS) model. The DRLS model uses an edge-based active contour method to drive the level set function (LSF) to the desired boundary, and provides a simple and efficient narrowband implementation without re-initialization.

Let $\phi: \Omega \rightarrow \Re$ be a level set function defined on domain $\Omega$. An energy function $\varepsilon(\phi)$ is defined as:

$$
\varepsilon(\phi)=\beta R_{p}(\phi)+\varepsilon_{a x}(\phi)
$$


where $\beta>0$ is a constant and $R_{p}(\phi)$ is the level set regularization term, defined by

$$
R_{p}(\phi)=\int_{\Omega} p(|\nabla \phi|) d x
$$

where $p$ signifies an energy density function $p:[0, \infty) \rightarrow \mathfrak{R}$, defined as

$$
p(s)= \begin{cases}\frac{1}{2}(s-1)^{2}, & \text { if } s>1 \\ \frac{1}{(2 \pi)^{2}}(1-\cos (2 \pi s)), & \text { otherwise }\end{cases}
$$

The minimization of the energy $\varepsilon(\phi)$ can be achieved by solving a level set evolution equation. For a LSF, an external energy function is defined by

$$
\varepsilon_{\text {cat }}(\phi)=\lambda L_{g}(\phi)+\alpha A_{g}(\phi),
$$

where $\lambda$ and $\alpha$ are the coefficient of the length term $L_{s}(\phi)$ and area term $A_{s}(\phi)$, which is given by

$$
L_{g}(\phi)=\int g \delta_{s}(\phi)|\nabla \phi| d x
$$

And

$$
A_{g}(\phi)=\int g H(-\phi) d x
$$

where $g \in[0,1)$ is an edge indicator function given by

$$
g=\frac{1}{1+\left|\nabla G_{\sigma} \cdot I\right|^{2}},
$$

where $G_{\sigma}$ is a Gaussian kernel with standard deviation $\sigma$, and $I$ is the input image. The Dirac delta function $\delta_{\varepsilon}$ and Heaviside function $H_{\varepsilon}$ in Eqs. (5)and (6) are approximated by the following smooth function $\delta_{\varepsilon}$ and $H_{\varepsilon}$, respectively, as in many level set methods:

$$
\delta_{\varepsilon}(s)= \begin{cases}\frac{1}{2 \varepsilon}\left[1+\cos \left(\frac{\pi t}{\varepsilon}\right)\right], & \text { if }|t| \leq \varepsilon \\ 0, & \text { otherwise }\end{cases}
$$

and

$$
H_{\varepsilon}(s)= \begin{cases}\frac{1}{2}\left(1+\frac{t}{\varepsilon}+\frac{1}{\pi} \sin \left(\frac{\pi t}{\varepsilon}\right)\right), & \text { if }|t| \leq \varepsilon \\ 0, & \text { if } t<-\varepsilon \\ 1, & \text { if } t>\varepsilon\end{cases}
$$


where $\mathcal{E}$ is a constant, typically set to 1 .5.The length term $L_{g}(\phi)$ was first introduced by Caselleset al.[24]in their proposed geodesic active contour (GAC) model. $L_{g}(\phi)$ computes the line integral of the function galong the zero level contour of $\phi$, which is minimized when the zero level set of $\phi$ is located at the object boundaries which in turn keeps the curve smooth during the deformation.The area term $A_{g}(\phi)$ calculates the weighted area inside the evolving contour. It is introduced to speed up the motion of the zero level contourwhen the contour is far away from the desired object boundaries and slow down the expanding and shrinking of the zero level contour when it arrives at object boundaries where $g$ is smaller. $A_{g}(\phi)$ represents a balloon forces in which the sign of $\alpha$ controls the direction of the level set evolution (shrinking or expanding).The level set evolution equation in the DRLS model is defined by:

$$
\frac{\partial \phi}{\partial t}=\beta \cdot \operatorname{div}\left(d_{p}(|\nabla \phi|) \nabla \phi\right)+\delta(\phi) \cdot \lambda \cdot \operatorname{div}\left(g \frac{\nabla \not \partial}{|\nabla \phi|}\right)+\delta(\phi) \cdot \alpha \cdot g
$$

The problem with the DRLS model in the case of liver segmentation is that the curve will evolve and deviate from the liver boundary in the region with weak or without edges. In this contribution, we will modify the distance regularization level set method [23] (DRLSM) by adding a new balloon force to guide the evolution process and discourage the evolving contour from leaking at a region with a weak or without an edges and from going far from the liver boundary.

\section{THE PROPOSED MODEL}

In this paper, we propose a new balloon force that controls the direction of the evolution and slows down the evolving contour at weak or blurred edges. Since the liver has a very similar intensity with its adjacent organs, this could easily result in over and/or under segmentation results. The DRLS model does not perform well with liver segmentation. We will modify the DRLS model to segment the liver contour in each 2D slice by using a new balloon force that controls the direction of the evolution and slows down the evolution process in the region with weak or without edges, which subsequently discourage the evolving contour from leaking at a region with a weak or without an edge and from deviating from the liver boundary. Our balloon term will be built using the probability density function. The methodology encompasses steps described in the following sections.

\subsection{PRE-PROCESSING}

The intensity distribution of the liver is irregular due to noises, so liver segmentation without preprocessing is difficult. A smoothing step, in theory, would make the intensity distribution less variable. In our work, a Gaussian filter is used as a smoothing step.

\subsection{SEGMENTATION OF THE REFERENCE SLICE}

This step is the most important step in our 3D liver segmentation method. The segmented liver contour will be the initial contour for the adjacent slice so the segmentation result should be 
accurate. The starting slice or the reference slice can be selected as a middle or the largest slice of the liver volume. In this contribution we used the Active Shape Model (ASM) [25] to segment the reference slice.

\subsection{D SLICE BASED PROPAGATION APPROACH}

Since the variation of shape and intensity between the adjacent slices are very small we can use these information from the previous slice to segment the next slice. In our method we compute the mean intensity $\mu$ and the variance $\sigma$ of the segmented slice. According to [26],about $98 \%$ of liver pixel is located in $[\mu-3 \sigma, \mu+3 \sigma]$.Generating an evolution region by expanding the previous segmented slice by a number of pixels and computing the probability density function inside this region using the following equations:

$$
B(X)= \begin{cases}\frac{1}{\sigma \sqrt{2 \pi}} e^{-\frac{1}{2}\left(\frac{x-\mu}{\sigma}\right)^{2}}, & \text { if } x \in[\mu-3 \sigma, \mu+3 \sigma] \\ 0, & \text { otherwise }\end{cases}
$$

We then shrink the previous segmented slice and use it as the initial contour for its adjacent slices in both directions.

\subsection{MODIFIED DRLS MODEL}

Since the liver boundary to be segmented is not far from the contour propagated from the previous slice, a shape and intensity prior information will discourage the evolving contour from leaking at a region with a weak edge or without an edge. We have modified the DRLSM by adding the probability density energy term to the evolution equation and use it as a balloon forces to control the direction and the speed of the evolution process

$$
E(\phi)=\rho \cdot R_{P}(\phi)+\lambda \cdot L_{g}(\phi)+\alpha \cdot B(\phi)
$$

where $\rho, \lambda$ and $\alpha$ are the coefficients of regularization term, length term, and probability term, respectively. This energy functional can be minimized by solving the following gradient flow:

$$
\frac{\partial \phi}{\partial t}=\rho \cdot \operatorname{div}\left(d_{p}(|\nabla \phi|) \nabla \phi\right)+\delta_{s}(\phi) \cdot \lambda \cdot \operatorname{div}\left(g \frac{\nabla \phi}{|\nabla \phi|}\right)+\delta_{s}(\phi) \cdot \alpha \cdot B(\phi)
$$

The above procedure is repeated until the contours in all $2 \mathrm{D}$ slices of the $3 \mathrm{D}$ image are segmented. A 3D liver surface is reconstructed from the contours segmented from all 2D slices.

\section{RESULT AND DISCUSSION}

In the DRLS model, two segmentation stages are applied. The first stage is for evolving the contour in the direction of the object boundary, speeding up the evolution process when the evolving contour is far from the object boundary and slowing down the evolution process when the evolving contour is close to the object boundary. The second stage concerns with the 
refinement of the segmentation results. In each experiment, we selected values of $\rho, \lambda$ and $\alpha$ to be $0.02,5$ and -1 for the first stage and $0.02,5$ and 0 for the second stage, respectively. The zero level set is initialized as a binary function and evolves according to the evolution equation, Eq. (13) for our model and Eq. (10) for the DRLS model.

Figures 1 and 2 present segmentation results of the DRLS model and the proposed model in a liver CT slice. Our model performs well and gives a satisfactory result comparing to the DRLS model. The DRLS model fails to segment the liver boundary and the evolving contour leaks from the region with weak edges. Our balloon force slows down the evolution process close to the liver boundary and stops the evolving contour from going far in the region with weak or without edges. Comparing with the DRLS model, our model is more effective in dealing with over segmentation problem.
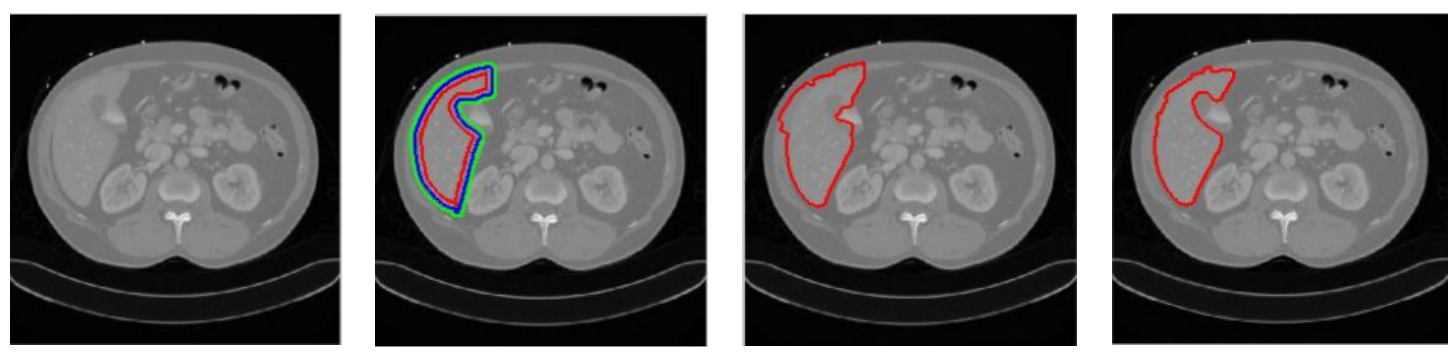

Figure1. Comparisons of liver segmentation result between the DRLS model and the proposed method. The first image shows the liver slice in a CT scan; the second image shows the previous segment contour in blue, evolving region in green and the initial contour in red. The third image shows the final segmentation result of the DRLS model and the fourth image shows the final segmentation result with our proposed method.
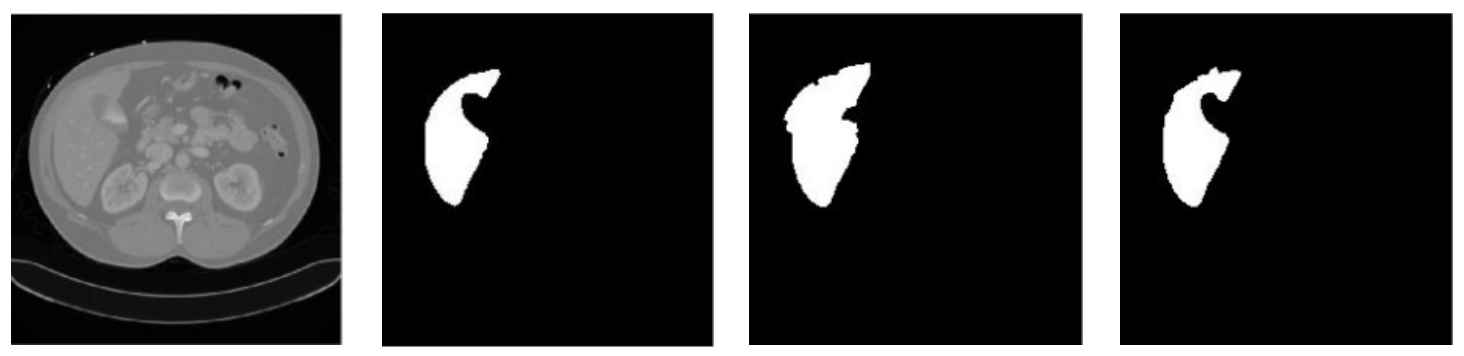

Figure2. Comparisons of liver segmentation result between the DRLS model and the proposed method. The first image shows the liver slice in a CT scan; the second image shows the ground truth segmented manually by a radiologist. The third image shows the final segmentation result of the DRLS model and the fourth image shows the final segmentation result with our proposed method.

Figure 3 shows some examples of liver extraction results based on our proposed method. We tested our model on a liver dataset containing 10 volumes of abdominal CT images. Each volume has 64 slices and the size of each slice is 512x512pixels. Each slice in the dataset is provided with corresponding ground truth segmented manually by a radiologist. The model deals very well with over segmentation problem. Our model can handle the over segmentation problems very well in comparison with the DRLS model that is not able to carry out this task. 

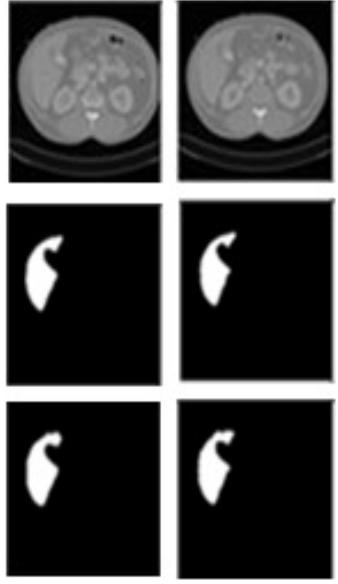
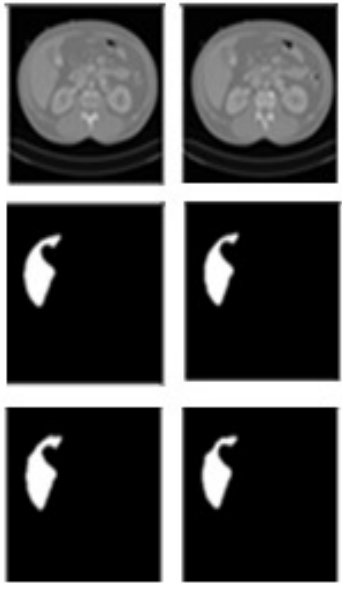
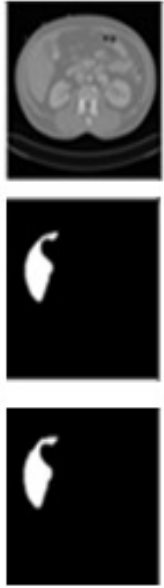
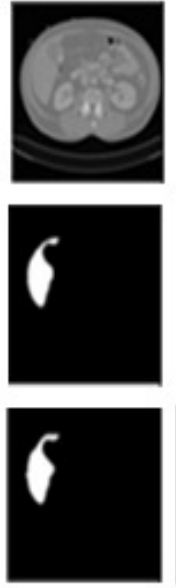
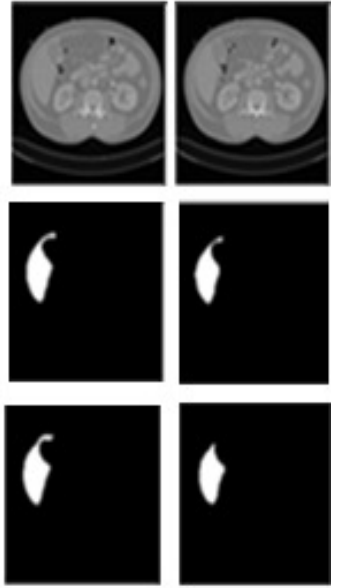

Figure 3.The experimental result of our proposed method on a sequence of liver slices for one person. The first row shows the liver slice in a CT scan; the second row shows the ground truth segmented manually by the radiologist. The third row shows the final segmentation result of our proposed method.

\section{CONCLUSION}

A novel balloon force method is presented herein. The main merits of this approach lies in its ability to guide the direction of the evolving contour and slows down the evolving contour in regions that are associated with weak or without edges and discourages the evolving contour from going far away from the liver boundary or from leaking at a region that has a weak edge, or does not have an edge. The model utilises a modified Distance Regularized Level Set (DRLS) model. The experimental results demonstrate that the method can achieve a satisfactory result. Our model proves to be more effective in dealing with over segmentation problems if compared with the DRLS model.

\section{REFERENCE}

[1] Luo, S., Li, X. \& Li, J. (2014) "Review on the Methods of Automatic Liver Segmentation from Abdominal Images",Journal of Computer and Communications. Vol. 2, No.2, pp 1-7.

[2] Adams, R. \& Bischof, L. (1994) "Seeded region growing",IEEE Transactions onPattern Analysis and Machine Intelligence, Vol. 16, No.6, pp 641-647.

[3] Beck, A. \& Aurich, V. (2007) "Hepatux-a semiautomatic liver segmentation system",3D Segmentation in The Clinic: A Grand Challenge. pp 225-233.

[4] Pohle, R.,\& Toennies, K. D. (2001)Segmentation of medical images using adaptive region growing. in Medical Imaging International Society for Optics and Photonics.

[5] Mortelé, K. J., Cantisani, V., Troisi, R., De Hemptinne, B.,\& Silverman, S. G. (2003) "Preoperative liver donor evaluation: imaging and pitfalls",Liver Transplantation. Vol. 9, No.9, pp S6-S14.

[6] Kumar, S., Moni, R.,\& Rajeesh, J. (2013) "Automatic liver and lesion segmentation: a primary step in diagnosis of liver diseases",Signal, Image and Video Processing. Vol. 7, No.1, pp 163-172.

[7] Platero, C., Poncela, J. M., Gonzalez, P., Tobar, M. C., Sanguino, J., Asensio, G. \& Santos, E. (2008)Liver segmentation for hepatic lesions detection and characterisation. in 5th IEEE International Symposium onBiomedical Imaging: From Nano to Macro, IEEE.

[8] Oliveira, D.B., Feitosa, R. Q. \& Correia, M. M. (2009)Liver Segmentation using Level Sets and Genetic Algorithms. in VISAPP (2). 
[9] Yang, H., Wang, Y., Yang, J.,\& Liu, Y. (2010)A novel graph cuts based liver segmentation method. in International Conference onMedical Image Analysis and Clinical Applications (MIACA), IEEE.

[10] Chen, Y.-W., Tsubokawa, K.,. \& Foruzan, A. H., (2010)Liver segmentation from low contrast open MR scans using k-means clustering and graph-cuts, in Advances in Neural Networks-ISNN 2010, Springer. pp 162-169.

[11] Foruzan, A. H., Yen-Wei, C., Zoroofi, R. A., Furukawa, A., Masatoshi, H. \& Tomiyama, N. (2013) "Segmentation of Liver in Low-Contrast Images Using K-Means Clustering and Geodesic Active Contour Algorithms",IEICE Transaction on Information and Systems, Vol. 96, No.4, pp 798-807.

[12] Liu, J. \& Udupa, J. K. (2009) "Oriented active shape models",IEEE Transactions onMedical Imaging, Vol. 28, No.4, pp 571-584.

[13] Heimann, T., Wolf, I. \& Meinzer, H.P. (2006)Active shape models for a fully automated 3D segmentation of the liver-an evaluation on clinical data, in Medical Image Computing and ComputerAssisted Intervention-MICCAI 2006, Springer. pp 41-48.

[14] Erdt, M., Steger, S., Kirschner, M. \& Wesarg, S. (2010)Fast automatic liver segmentation combining learned shape priors with observed shape deviation. in IEEE 23rd International Symposium onComputer-Based Medical Systems (CBMS), IEEE.

[15] Badakhshannoory, H. \& Saeedi, P. (2011) "A model-based validation scheme for organ segmentation in CT scan volumes",IEEE Transactions onBiomedical Engineering, Vol. 58, No.9, pp 2681-2693.

[16] Huang, W., Tan, Z., Lin, Z., Huang, G., Zhou, J., Chui, C., Su, Y. \& Chang, S. (2012)A semiautomatic approach to the segmentation of liver parenchyma from 3D CT images with Extreme Learning Machine. in Annual International Conference of the IEEE Engineering in Medicine and Biology Society (EMBC), IEEE.

[17] Danciu, M., Gordan, M., Florea, C. \& Vlaicu, A. (2012)3D DCT supervised segmentation applied on liver volumes. in 35th International Conference onTelecommunications and Signal Processing (TSP), IEEE.

[18] Luo, S., Li, X. \& Li, J. (2013) "Improvement of Liver Segmentation by Combining High Order Statistical Texture Features with Anatomical Structural Features",Engineering. Vol. 5, No.5, pp 6772.

[19]Luo, S., Hu, Q., He, X. , Li, J. , Jin, J. S. \& Park, M. (2009)Automatic liver parenchyma segmentation from abdominal CT images using support vector machines. in ICME International Conference onComplex Medical Engineering, IEEE.

[20] Xu, C., Pham, D. \& Prince, J. (2000)Medical Image Segmentation Using Deformable Models, in SPIE Handbook on Medical Imaging J.M. Fitzpatrick and M. Sonka, Editors, pp 129-174.

[21] Altarawneh, N. M., Luo, S., Regan, B., Sun, C. \& Jia, F. (2014) "Global threshold and region-based active contour model for accurate image segmentation",Signal \& Image Processing: An International Journal (SIPIJ). Vol.5, No.3, pp 1-11.

[22] Altarawneh, N. M., Luo, S., Regan, B., Sun, C. (2014) "A novel global threshold-based active contour model",Second International Conference on Signal, Image Processing and Pattern Recognition(SIPP),pp 245-254.

[23] Li, C., Xu, C., Gui, C. \& Fox, M. D. (2010) "Distance regularized level set evolution and its application to image segmentation",IEEE Transactions onImage Processing, Vol. 19, No.12, pp 3243-3254.

[24] Caselles, V., Kimmel, R. \& Sapiro, G. (1997) "Geodesic active contours",International Journal of Computer Vision. Vol. 22, No.1, pp 61-79.

[25] Van Ginneken, B., Frangi, A. F., Staal, J. J., Ter Haar Romeny, B. M. \& Viergever, M. A. (2002) "Active shape model segmentation with optimal features",IEEE Transactions onMedical Imaging, Vol. 21, No.8, pp 924-933.

[26] Li, X., Luo, S. \& Li, J. (2013) "Liver Segmentation from CT Image Using Fuzzy Clustering and Level Set",Journal of Signal and Information Processing. Vol. 4, No.3, pp 36-42. 


\section{AUTHORS}

Nuseiba Altarawneh is a $\mathrm{PhD}$ student at the University of Newcastle, Australia. Her research interests include computer vision, image analysis, and pattern recognition. She obtained the M.E. degree in Computer Science from the University of Jordan in 2009.

Dr. Suhuai Luo received the $\mathrm{PhD}$ degree in Electrical Engineering from the University of Sydney, Australia in 1995. From 1995 to 2004, he worked as a senior research scientist with the Commonwealth Scientific and Industrial Research Organization Australia and the Bioinformatics Institute Singapore. He is currently a senior lecturer with the University of Newcastle, Australia. His research interest is in information technology and multimedia, including health informatics, machine learning, image processing, computer vision, and Internet-oriented IT applications.

Dr. Brian Regan is a senior lecturer in IT at the University of Newcastle. He is part of the Applied Informatics Research Group (AIR) with interests in health informatics, visualization and development methodologies.

Dr. Changming Sun received the $\mathrm{PhD}$ degree in the area of computer vision from Imperial College London in 1992. Then, he joined CSIRO Computational Informatics, Australia, where he is currently a principal research scientist carrying out research and working on applied projects. His research interests include computer vision, image analysis, and pattern recognition. He has served on the program/organizing committees of various international conferences. He is an Associate Editor for EURASIP Journal on Image and Video Processing, a Springer One journal.
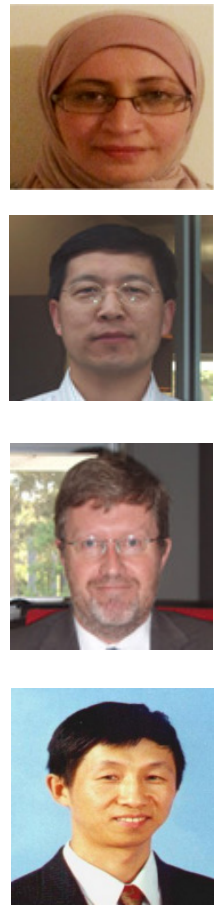